\title{
New Microwave Sensing System for Blade Tip Clearance Measurement in Gas Turbines
}

\author{
Maddalena Violetti and Anja K. Skrivervik \\ Laboratoire d'Electromagnétisme et d'Acoustique (LEMA), \\ École Polytechnique Fdrale de Lausanne, \\ CH-1015 Ecublens, Switzerland \\ Email: maddalena.violetti@epfl.ch
}

\author{
Qin Xu and Michaël Hafner \\ Meggitt Sensing Systems \\ CH-1701 Fribourg, Switzerland \\ Email: qin.xu@ch.meggitt.com
}

\begin{abstract}
Real-time clearance control in turbine engines allows improving their efficiency and safety, by enabling prognostics and optimized condition-based maintenance in the turbine hot section. This paper presents a novel $24 \mathrm{GHz}$ microwave sensing system for real-time blade tip clearance monitoring suitable for small-size turbines (aero-engines and aero-derivatives). The system principle of operation is similar to the short range radar technique and employs high temperature resistant circular waveguide resonator probes mounted into the shell of the turbine. Probes concept was proven through simulation and prototypes where built and tested, showing agreement of results. The system was validated in laboratory, where the harsh turbine environment was reproduced on dedicated test rigs. Finally, the system was tested on a real engine, showing its suitability for real-time blade tip measurement.
\end{abstract}

\section{INTRODUCTION}

Control of blade-tip clearance in the high pressure compressor and turbine sections of gas turbine engines is essential for improved efficiency, reliability and cost effective maintenance. It has been reported in literature that a $0.0254 \mathrm{~mm}$ of tip clearance reduction can contribute as much as an additional $1 \%$ in improved efficiency [1]. Tip clearances should be designed to be as small as possible, yet still not rub the case as the engine goes through its operating envelope, eventually causing disintegration [2].

This is true for both land based and aero engines, however tip sealing is more challenging for the latter due to the frequency of change of engine operating modes as well as inertial and aerodynamics loads during flight. It was demonstrated that significant clearance variations occur during a common commercial flight profile [3].

Due to the difficulty in getting tip clearance sensors to provide accurate and reliable data for an extended period of time, the design of tip clearances can often be conservative.

Active real-time clearance control systems can adjust the tip clearances dynamically for the current engine operation envelope providing constant engine performance and avoiding frequent and costly overhauls [4].

To date, many sensing strategies are used for the purpose of rotor and blade health monitoring [5], based on optics, laser, capacitance, eddy currents and microwaves [6], [7].

In general, measuring probes mounted in turbines must be capable of operating for an extended period of time in the

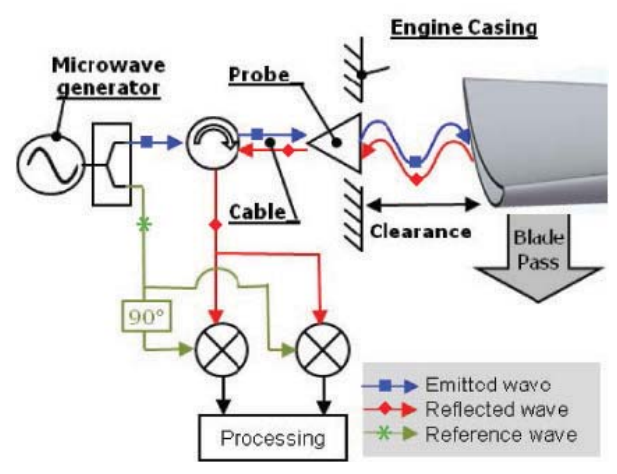

Fig. 1. $24 \mathrm{GHz}$ Meggitt tip clearance measurement system architecture.

presence of vibration, corrosive gases and large temperature range, typically from $-30^{\circ} \mathrm{C}$ to over $1300^{\circ} \mathrm{C}$ depending on sensor location within the turbine. This harsh environment of operation limits long-term use of most candidate technologies. In addition, sensors should be hermetically sealed and compact in order to avoid perturbing the performance of the turbine. Among the above-mentioned blade tip monitoring techniques, microwave sensors are advantageous for their ability to operate at extremely high temperatures, unaffected by dirt and contaminants that may prevent the long-term use of other available technologies in the harsh turbine environment.

In this paper we present a new $24 \mathrm{GHz}$ microwave sensing system (patent pending [8]), similar to a short-range radar and suitable for tip clearance measurement in small gas turbines (aero-derivatives and aero-engines). The tip clearance measurement system (shown in Fig. 1) uses miniature resonator probes mounted into the turbine case, in direct view of the passing blades.

The selected probe design consists of a resonant cylindrical open-ended waveguide cavity made of high temperature resistant materials, fed by a coaxial line and protected by a ceramic cap. This technology represents a smart compromise between miniaturization and robustness of the probe.

The proposed design was validated through software simulation and prototypes were built and tested. Measurements show excellent agreement with simulated results.

In addition, environmental tests on isothermal aging, thermal 


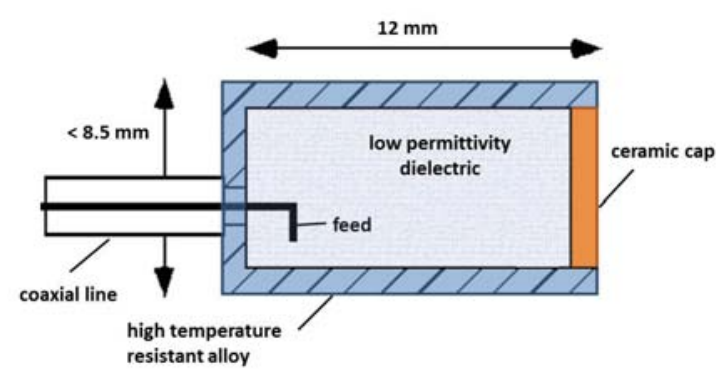

Fig. 2. Cut view of the $24 \mathrm{GHz}$ waveguide resonator probe (outer diameter $<8.5 \mathrm{~mm})$.

cycling up to $900^{\circ} \mathrm{C}$ and vibration were carried out with success. Finally, the system was successfully tested on aeroengine turbines for tip clearance measurement at a power load ranging from $1 \mathrm{MW}$ to $20 \mathrm{MW}$.

\section{Microwave System Overview}

The microwave high temperature blade tip clearance system presented in this paper (Fig. 1) works on principles that are similar to the short range radar technique [9].

It is composed of eight independent channels operating at $24 \mathrm{GHz}$. Each channel is composed of a microwave probe installed in the engine, a microwave cable and an electronics cards pair. The electronics cards are protected by a thermally regulated enclosure located away from the hot area of the engine. The microwave probes are connected to these electronics by means of high temperature resistant cables. Additionally to the microwave channels, the speed signal of the high pressure rotor is provided to the system for synchronization.

The raw signal acquisition scheme is based on periodic snapshot every second. Each snapshot catches exactly one rotor revolution by being synchronized with the rotor speed signal. The sensors are not making measurements in-between acquisition and during this time the signal processing is performed. Therefore, each sensor provides a tip clearance update of the rotor blades every second.

\section{A. Sensor description}

The measurement system employs high temperature resistant microwave probes which are mounted in the case of the turbine, such that they have a direct view of the blade tips. The selected probe design consists of an open-ended circular waveguide resonator fed by a coaxial line and protected by a ceramic cap, as shown in Fig. 2. This technology allows to achieve great miniaturization while the sensor structure is very robust. The small probe outer diameter $(8.5 \mathrm{~mm})$ minimizes the probe intrusion inside the engine.

The sensors send a continuous frequency swept microwave signal towards the target (i.e. the rotating turbine blades) and receive the reflected signal. The parameters of interest are both the amplitude and phase of the signal, in and out of resonance. At resonance the electromagnetic (EM) field is radiated by the sensor and interacts with the tip of the blade, while out of resonance the EM field is not able to detect the blade, yielding

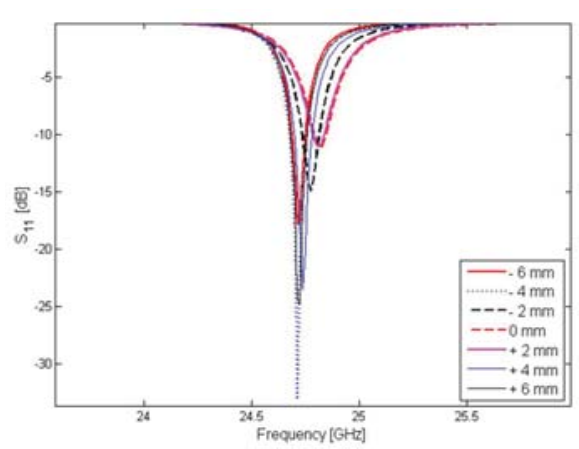

Fig. 3. Reflection coefficient (magnitude), showing the influence of a blade model sweeping by the probe tip $(0 \mathrm{~mm}=$ center $)$ at $1 \mathrm{~mm}$ clearance. Sweep direction is $90^{\circ} \mathrm{C}$ with respect to the feed plane.

a reference signal. From this information, the system is able to yield tip clearance, by means of a phase-based measurement principle.

\section{Probe Concept VAlidation}

The probe concept was investigated through software simulation, in order to prove the reliability and robustness of selected technology under harsh operating conditions. Simulations were aimed to study the influence of relevant geometrical features (e.g. feed position and shape, cap thickness, behavior of materials vs. temperature) and of the blade-probe interaction.

For the specific application, the $24 \mathrm{GHz}$ waveguide cavity probe should allow for great mechanical stability. The sensor should have a single resonance within the $600 \mathrm{MHz}$ band of interest. The resonance should be sharp and only weakly temperature dependent.

The simulated model presents a single sharp resonance, in excellent agreement with measured results.

The large temperature variation of the turbine environment was simulated by increasing the nominal permittivity value (at room temperature) of the ceramic cap by $30 \%$ with a step of 0.5 . The measured model shows a small shift of the reflection coefficient due to temperature change, which meets the system bandwidth requirements.

Finally, the blade-probe interaction was studied by sweeping a blade model in front of the probe tip for different combinations of blade-to-probe clearance and orientation. Results show the sensitivity of probe response in the presence of the blade, demonstrating its usefulness for the purpose of blade tip detection. As a sample result, Fig. 3 shows the simulated reflection coefficient (magnitude) of the probe in the presence of a blade model sweeping by the probe tip $(0 \mathrm{~mm}=$ center $)$ at $1 \mathrm{~mm}$ clearance. The sweep direction is $90^{\circ} \mathrm{C}$ with respect to the feed plane.

\section{LABORATORY TESTS}

Prototypes of the optimized model (see Fig. 4) were built and successfully tested.

The system was validated in laboratory, where the harsh turbine environment (with vibrations and temperatures exceeding 


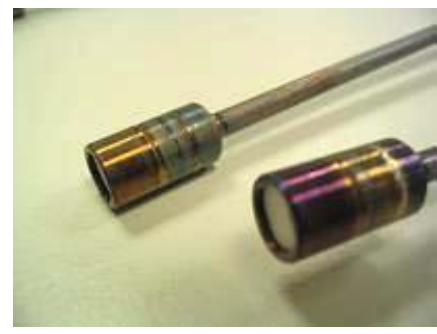

Fig. 4. Two of the built prototypes.

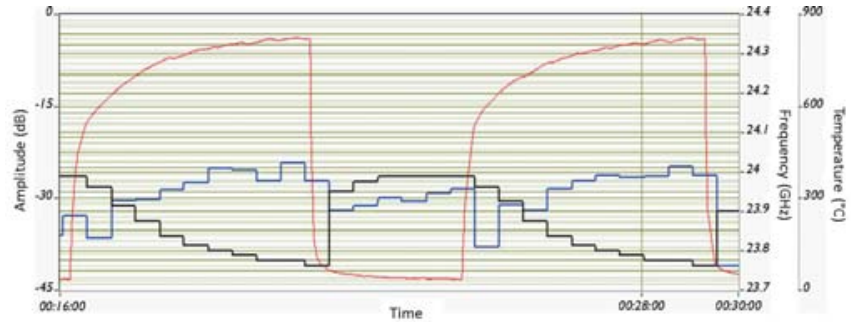

Fig. 5. Thermal test results. Thermal gradient applied to the probe (red). Resonance depth (blue) and center frequency (black) show a minimum shift.

$900^{\circ} \mathrm{C}$ ) was reproduced on special test rigs.

Thermal tests comprised isothermal aging at $900^{\circ} \mathrm{C}$ for over 4500 hours and thermal cycling from $20^{\circ} \mathrm{C}$ to $900^{\circ} \mathrm{C}$ with cooling air for more than 1200 cycles. Frequency response remains stable towards the applied thermal gradient (as shown in Fig. 5) demonstrated the suitability of probes for operation in the presence of high temperature extremes.

Vibration tests were carried out using a dedicated shaking test bed. Probes endured 5 hours random and sinusoidal vibration at low frequency (up to $2 \mathrm{kHz}$ ) and 3 hours sinusoidal vibration at high frequency $(2-10 \mathrm{kHz})$. The frequency notch was monitored before and after the test showing minimum change.

In order to test blade detection capability, probes axial position (distance from the leading edge of the blade) and orientation (on-axis rotation) was randomly changed. At the optimal position (shown in Fig. 6), results show good linearity between real and measured clearance. Finally, individual probe calibration was performed.

The error due to rotor axial shift was estimated to be within $\pm 0.1 \mathrm{~mm}$. Probe calibration curves present some offset differences which are due to probe manufacturing variability.

\section{FIELD TESTS}

The full system was tested on a real gas turbine engine, in order to assess its suitability for the envisaged application.

The microwave probes were mounted in the high pressure turbine stage of a DM80 engine (25MW rated output power) with clearance being monitored during engine operation.

After calibration at the optimum position, results showed linearity of measured vs. real clearance and the system ability to measure individual clearances over the engine operation modes (i.e. engine power sweeping, hot restart and activation

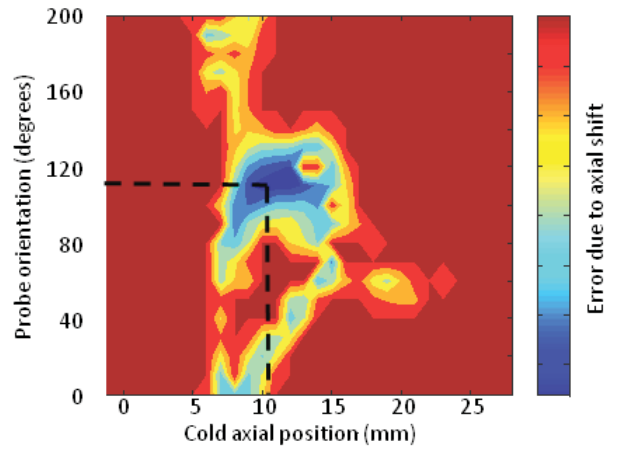

Fig. 6. Measurement error due to axial shift during engine operation for different combinations of the probe orientation and cold axial position. The optimum probe installation position is indicated by the dashed lines.

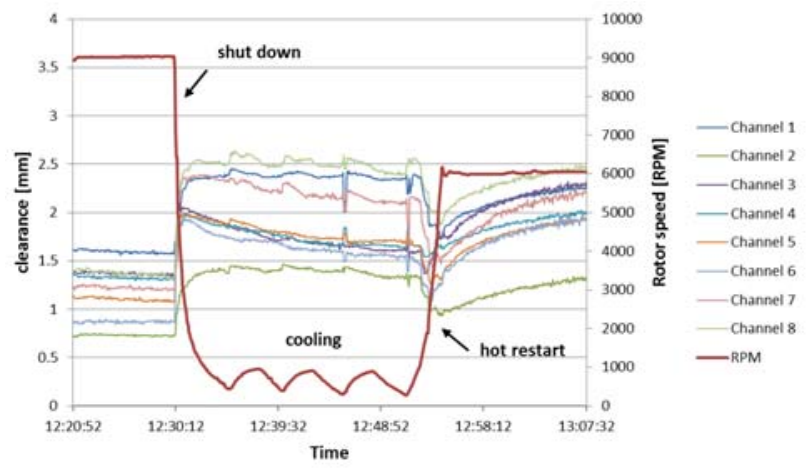

Fig. 7. Blade tip clearance measured in real-time by 8 probes (= channels) during engine shut-down, cooling and hot restart.

of a casing cooling system).

As a sample result, Fig 7 shows real-time blade tip clearance measurement by 8 probes (= channels) during engine shutdown, cooling and hot restart. The microwave system was able to measure clearance correctly during the different phases, as the measured values are consistent from probe to probe and with respect to the expected engine behavior.

\section{CONCLUSION}

The new microwave sensing system was proven to be suitable for operation inside the turbine engine harsh environment. Clearance measurements yielded by the performed tests are within the range of estimated values and represent an essential tool for test engineers. Tests campaigns are anyway recommended to confirm the long term stability of the system and durability of probes towards aging.

\section{ACKNOWLEDGMENT}

This work was supported by the Swiss Federal Government CTI (Commission for Technology and Innovation) grant $\mathrm{n}$ 9301.1 PFNM-NM.

The authors would like to thank Gérald Egger and Jon Geisheimer of Meggitt Sensing Systems for their support. 


\section{REFERENCES}

[1] M. W. Wiseman and T.-H. Guo, "An investigation of life extending control technoques for gas turbine engines," in Proc. American Control Conference, June 25-27, Arlington, VA 2001.

[2] N. Goel, A. Kumar, V. Narasimhan, A. Nayak, and A. Srivastava, "Health risk assessment and prognosis of gas turbine blades by simulation and statistical methods," in Proc. Canadian Conference on Electrical and Computer Engineering, May 4-7, 2008.

[3] S. B. Lattime and B. M. Steinetz, "Turbine engine clearance control systems: Current practices and future directions," in 38th AIAA Joint Propulsion Conference and Exhibit, July 7-10, Indianapolis, IN, 2002.

[4] J. L. Geisheimer and T. Holst, "Metrology considerations for calibrating turbine tip clearance sensors," in Proc. XIX Biannual Symposium on Measuring Techniques in Turbomachinery Transonic and Supersonic Flow in Cascades and Turbomachines, Rhode-St-Genèse, Belgium April 7-8, 2007.

[5] A. Von Flotow, M. Mercadal, and P. Tappert, "Health monitoring and prognostics of blades and disks with blade tip sensors," in Proc. of the 2000 IEEE Aerospace Conference, 2000.

[6] M. Violetti, J.-F. Zürcher, J. Geisheimer, and A. K. Skrivervik, "Design of antenna based sensors for blade tip clearance measurement in gas turbines," in Proc. 4th European Conference on Antennas and Propagation, 12-16 April, Barcelona, Spain 2010.

[7] A. B. Vakhtin, S.-J. Chen, and S. M. Massick, "Optical probe for monitoring blade tip clearance," in 47th AIAA Aerospace Sciences Meeting Including The New Horizons Forum and Aerospace Exposition, January 5-8, Orlando, FL, 2009.

[8] M. Violetti, A. K. Skrivervik, Q. Xu, J. Geisheimer, and G. Egger, "Device and method for monitoring rotor blades of a turbine," European Patent Application No. 11181622, Sept. 16, 2011.

[9] M. R. Woike, J. W. Roeder, C. E. Hughes, and T. J. Bencic, "Testing of a microwave blade tip clearance sensor at the NASA Glenn research center," in 47th AIAA Aerospace Sciences Meeeting, Orlando, Florida 5-8 January, 2009. 